(C)2009 IEEE. Personal use of this material is permitted. However, permission to reprint/republish this material for advertising or promotional purposes or for creating new collective works for resale or redistribution to servers or lists, or to reuse any copyrighted component of this work in other works must be obtained from the IEEE. 


\title{
A New Significant Area: Emotion Detection in E-learning Using Opinion Mining Techniques
}

\author{
Haji H. BINALI, Chen WU, Vidyasagar POTDAR
}

${ }^{1}$ Haji H. Binali, Digital Ecosystems and Business Intelligence Institute, Curtin University of Technology, Perth, Australia, email: h.binali@postgrad.curtin.edu.au

${ }^{2,3}$ Chen Wu and Vidyasagar Potdar, Digital Ecosystems and Business Intelligence Institute,

Curtin University of Technology, Perth, Australia, email: (chen,v).author@curtin.edu.au

\begin{abstract}
E-learning has sprung up much interest in corporations, educational institutions and individuals alike. Recently, it has been discovered that emotion can affect the elearning experience. However, understanding the emotional reaction of a student in a complicated learning environment is a mind boggling task. By detecting intense emotional experiences being exhibited by students, we intend to detect fluctuations in emotion as learning progresses. To achieve this, we present a conceptual emotion detection and analysis system for e-learning using opinion mining techniques.
\end{abstract}

Keywords — E-learning, opinion mining, emotion detection

\section{INTRODUCTION}

E-learning has sprung up much interest in corporations, educational institutions and individuals alike. It commonly refers to teaching efforts propagated through the use of computers in an effort to impart knowledge in a non traditional classroom environment. Recent studies have revealed that emotion can affect the e-learning experience.

In general, emotion has to do with how one feels. This feeling, if positive is believed to have a productive effect on the individual. However, feelings of a negative nature seem to impact negatively on the individual's learning experience. Obviously, the topic of emotions goes far beyond this simple definition and it is especially hard to detect in an elearning environment. In this paper, we propose to use opinion mining methods to address this issue.

Opinion mining is a field that deals with extracting opinions from user generated content and then providing it in a more useful format. Such formats include but are not limited to, the use of graphs and avatars. Anything that prevents one from engaging in the monotony of reading, viewing or listening to a large amount of information would be highly regarded as being a useful format. In our research, we will be exploring a course conducted at a university that requires students to submit weekly reviews of their participation. We aim to extract and analyse emotional content from online journals which will then be presented to the lecturer to assist in advancing pedagogy.

This paper is organized in the following sections. Sections II - Section IV presents a literature review of elearning, emotion and opinion mining. Section V proposes a new area of research for emotion detection and extraction in text. Section VI discusses a conceptual model, its contri- bution and significance in the research literature. We conclude the paper with Section VII.

\section{E-LEARNING}

The presentation of material to be learned in the online environment provides evidence that teaching is taking place; however, this is not the case when it comes to learning [1]. The fact that material has been presented online does not imply that students will learn in the required manner. Research shows that most e-learning applications disregard learning methods employed by people [1].

There are several e-learning issues to be dealt with, such as the involvement of peers in online discussions [2], emotional reactions towards a given task [3, 4]and the user opinions and their evaluations of services [5] like courseware and teaching staff. Goodman et al [2] present an example of how an agent can be used to encourage group collaboration by directing the questions of the discussion where the students appear to be stuck. Being stuck can lead students to becoming frustrated [6]. Burleson and Picard [7]propose the intervention of an automated peer companion to minimise discouragement or disappointment as a means to maintaining motivation towards a given task.

Intense emotional experiences and a lack of time management skills have been reported by e-learners[8]. This comes as no surprise given the difference in the learning paradigms of digital native learners and teaching paradigms employed by digital immigrant teachers[9]. Digital native is a term that is synonymous with N(.Net)-Gen or D (Digital)Gen, which refers to today's students born in the age of the internet, computers, video games and cell phones[10]. Digital immigrants refers to individuals who have adopted the technologies of the digital natives[10]. For example, the former prefer "processing pictures, sounds and video"[9]. Whereas the latter prefer "to provide text before pictures, sounds and video"[9]. Furthermore, digital immigrant teachers prefer " to teach to the curriculum guide and standardised tests"[9] in contrast to digital native learners who prefer " learning that is relevant, instantly useful and fun"[9]. As illustrated by the preceding examples, this difference in learning and teaching paradigm can affect the mental state of e-learners who may be lead astray from attaining intended objectives.

In face-to-face class, instructors can detect facial expressions of students but in an online environment, students need to establish an online presence and the instructors need to be able to pick up on this [11]. An online presence can be established by engaging in collaborative exercises like 
peer discussions and other online activities. The need to prepare students for e-learning is expressed in [16]. It was discovered that a group of MSc students in information systems were uncomfortable with posting comments online. This demonstrates that technical competence does not necessarily imply online learning competence in terms of emotional maturity.

A summary of prevalent e-learning issues that have been identified include technical problems in applications like files not being attached, hardware problems, lack of computer skills on the part of learners to handle given tasks , lack of preparedness of teachers to provide e-learning materials and ambiguous course objectives[3, 8,11$]$.

\section{EMOTION}

Previous studies have suggested that emotion is constantly associated with learning[3]. It is also understood that emotions can heavily influence the knowledge and overall goals of the students [17]. Hence, the significance of the emotional factor should not be neglected. However, understanding the emotional reaction of a student in a complicated learning environment is a challenging task. In fact not much work has been done to accurately measure the effect of emotion through the interaction between lecturers and students.

Education research has suggested that emotion has a significant impact on the learning process [18]. In fact, the connection between complex learning and emotions has been well documented in a number of related disciplines such as psychology [17], education [19], and computer science [20]. More recently, after reviewing 11 students, [3]concluded that emotion has played a central and essential role in the teaching and e-learning process.

Recognising the importance of emotion during learning is one thing. Detecting and utilising emotions of learners to augment the learning and teaching process is another. The AutoTutor research project has strived to achieve this goal. In this direction,[12] first investigated "the extent to which trained judges and untrained peers can accurately identify the affective states of learners who interact with AutoTutor". Based on this result, [21] then developed a metric to measure the possibility of "transitioning from an affective state at time $t 1$ to a subsequent affective state at time $t 2$ ". More recently, [22] investigated the reliability of detecting a learner's affective states using sensors with learners experience data.

While these studies have made some progress in detecting learners' emotions, they heavily rely on sensors in a laboratory setting, which may not be available in a real situation. To the best of our knowledge, little research has been done that is able to detect student emotion in a natural and spontaneous fashion without using experimental instruments.

\section{A. Emotions exhibited by learners}

Table 1 lists common emotions exhibited by learners which have been identified from the literature. "Sadness, happiness, anger, fear, disgust and surprise" [12] had been thoroughly investigated as having an impact on learning. In fact, [15] presented an e-learning system that recognises facial expressions related to these emotional states. However,

\begin{tabular}{|c|c|c|}
\hline Sadness & Interest & Melancholy \\
\hline Happiness & "stuck" & Wistfulness \\
\hline Anger & Boredom & Bewilderment \\
\hline Fear & Disappointment & Alienation \\
\hline Disgust & Discouragement & Euphoria \\
\hline Surprise & Anxiety & Ecstasy \\
\hline Confusion & Joy & $\begin{array}{l}\text { Fear- } \\
\text { confirmed }\end{array}$ \\
\hline Distress & Rage & Apprehension \\
\hline Frustration & Love & $\begin{array}{l}\text { Shame/ } \\
\text { Embarrass- } \\
\text { ment }\end{array}$ \\
\hline Flow/engagement & $\begin{array}{l}\text { Enthusiasm/ } \\
\text { Excitement }\end{array}$ & Satisfaction \\
\hline Pride & Grief & humiliation \\
\hline Delight & Relief & Stress \\
\hline
\end{tabular}

it was recently discovered by [12] that these emotions have minimal effect on learning. Contrary to this, "confusion, frustration, boredom, flow/ engagement, interest and being stuck" [12] have been found to have an enduring impact on learning. It has been noted by [7] that student motivation can be maintained when a state of stuck or fear is detected. They proposed intervention by AutoTutor (Intelligent Tutoring System) when a student is experiencing disappointment or discouragement to bolster self confidence by inducing motivation. Hara and Kling[11] have found out that confusion, anxiety, distress and frustration are attributed to unclear course objectives and a lack of prompt, clear communication via email by the instructors. This further supports the assertion by [9] on differences in the teaching and learning paradigms between digital native learners and digital immigrant teachers.

Although a large number of emotions have been identified by researchers $[13,14]$, thorough investigations are yet to be carried out to establish a "basic emotions set for learning" [14].

\section{OPINION MINING}

Opinion mining research is about the extraction and analysis of opinions expressed in various information sources like blogs and bulletin boards [23]. Terms synonymous with opinion mining are affective rating, sentiment analysis, sentiment extraction and sentiment classification[23]. According to [23], 'e-learning systems adoption by tertiary institutions is still in its early phases' but has gained popularity recently [5]. This new opportunity has inspired our current investigation. An introduction to opinion mining and an opinion mining framework that can be manipulated to work in an e-learning system was presented by[23].

Multiple techniques and principles have contributed towards research development in opinion mining such as appraisal theory, systematic functional linguistics, Natural Language Processing (NLP) and statistical schema matching. Major research has focused on term orientation analysis 
which assumes the non existence/availability of a lexical resource where terms have been tagged as either as being positive or negative thus requiring it to be auto generated [24]. In most cases, the lexical resource forms the basis for opinion mining.

Appraisal Theory is used to identify semantic orientation by treating words as attribute groups. Used in conjunction with the "bag of words" approach, a classification accuracy of $90.2 \%$ was achieved in an experiment by [25]. The "bag of words" approach selects frequently used words in a document to provide learning values. Different parts of text are then weighed and used in classification to give a thumbs up or thumbs down. On the other hand, semantic orientation classifies words into two groups, "good" and "bad". An overall rating is then given. Empirical results lead to the inference that these two major techniques do not cater for attitude expressions. An attempt is made to try and resolve this by using taxonomy of attitude types and semantic properties to form appraisal groups.

By using appraisal groups, the effect of modifiers (not at all, very) can be taken into account thereby improving the accuracy of sentiment analysis. Three Learning approaches are used. In the first, the algorithm places terms as either subjective or objective. The second classifier then marks the subjective terms as positive or negative. The second approach learns two classifiers. One classifier discriminates between terms that belong to the positive category and ones that belong to its complement. The third approach uses multi class classification with three categories; positive, negative and objective.

Corpus sentence level classification for automatic opinion mining has been achieved by evaluating the strength of opinions and emotions. Being cognizant of the broad vocabulary that can be used to express opinions, [26] infer that a system with fixed vocabulary would be inefficient to handle changing discourse. An attempt is made to address this by using syntactic clues and subjectivity clues. Experimental observations have led to the conclusion that polarity, an attribute of subjective language, can be a good indicator for the strength of subjective expressions. Opinion strength recognition is achieved through boosting, rule learning, and support vector regression. Apart from distinguishing between subjective and objective expressions, [26] have been able to classify nested clauses in all sentences in the corpus as well as distinguishing between weak, medium and strong subjectivity.

Research by [27] has enabled feature and item comparisons to be made intuitively. A comparison of products is carried out by algorithms and presented to the user in a graphical user interface for quick comparison. Opining mining is carried out using supervised (Naïve Bayes) and unsupervised learning (SO-PMI). The proposed system begins by performing an information search on a specific product, given as a Google search criterion. The structure is then analysed as a part of web data extraction. Feature extraction takes place during opinion mining and the values are stored in a database. These values are then used as input for rendering a graphic output to the user.

Statistical schema matching was used [28] to identify features used in a review. A schema that identifies and groups synonyms without semantic understanding is used for ma- chine learning purposes to try and identify the features used in a review. The innovation by [28] is the use of statistical methods in conjunction with machine learning to group features. Similar research involving statistical methods was used by [29].

Using reviews from computer and consumer electronics products, [29] discovered that reviews on movies, books and music tend to be more subjective, adding a layer of complexity to supervised learning. Multiple techniques such as Laplace smoothing, Naïve Bayes, Witten-Bell Method and Good Turing were used to award a final rating of reviews.

Currently, algorithms make the assumption that words used in opinion reviews are subjective. Furthermore, a methodology for establishing the reputation and reliability of the reviews is not being taken into account.

\section{EMERGING AREA OF SigNIFICANCE}

This paper will open up a new area by introducing opinion mining, a topic that has been recently explored in the business and computing domains, into the context of e-learning process. In particular, to provide a conceptual framework that can extract, analyse, and predict the emotion of learners (i.e. students) to assist the lecturer to make wise decisions in order to tailor the teaching methods and processes. Hence the central contribution of this research can be generalised to many teaching and learning activities at universities. That is, the learning and teaching methods and processes can be enhanced in certain ways when negative emotions are detected and minimized and when positive emotions are acknowledged, maximised, and utilised.

\section{A. Conceptual view of knowledge areas}

Our research is motivated by seeking an amalgamation of the knowledge areas shown in Fig.1. There is a plethora of research available for each of the main areas, opinion mining, e-learning and emotion detection. The motivation of our research was to find a common area, A, the convergence zone from which we could explore a new area of research. To the best of our knowledge, there is a paucity of literature in this area. The numbers 1-3 represent an intersection of the major fields in discussion. They are opinion mining and emotion detection (1), opinion mining and e-learning (2) and e-learning and emotion detection (3) respectively. The next paragraph explains how we intend to manipulate the three core knowledge areas.

E-learning domain knowledge gives us a perspective of the current state of affairs with regards to existing problems. Emotion detection literature will provide a clue on the emotion set exhibited by learners thereby paving a way forward for the annotation rules to be written and applied in the Gate infrastructure. The use of annotation rules is the means by which we intend to identify the emotions of students. From opinion mining research, we will explore ways to use the algorithms to help us in improving e-learning systems to make them engaging to the mind as well as motivating. One way of achieving this is by using annotation rules as explained previously. Annotation rules are no more than a way of marking/labelling the different words or characters that constitute a sentence. 


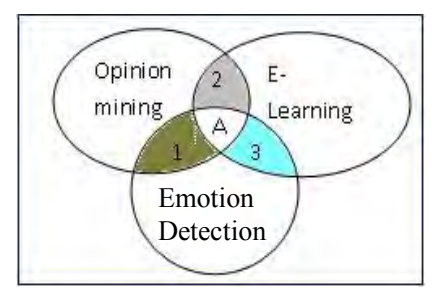

Fig.1 Conceptual view of knowledge areas

\section{B. Research objective statement}

Within the context of an e-learning system, the purpose of this research is:

- Detection of student emotion fluctuation as learning progresses

- How to identify struggling students

- How to automatically track attitudes and feelings in the course, faculty and peers

\section{CONCEPTUAL SOLUTION}

We envisage an e-learning system as shown in Fig.2. This is an extension of Fig. 1 to show the practical application of the knowledge areas. The core part of the research is to advance emotion detection in an e-learning system using opinion mining techniques. We will breakdown our discussion along three boundaries: Learning (corpus), Opinion mining \& Emotion Detection and output for e-learning advancement. In sections A-C, we explain in detail, the e-learning scenario.

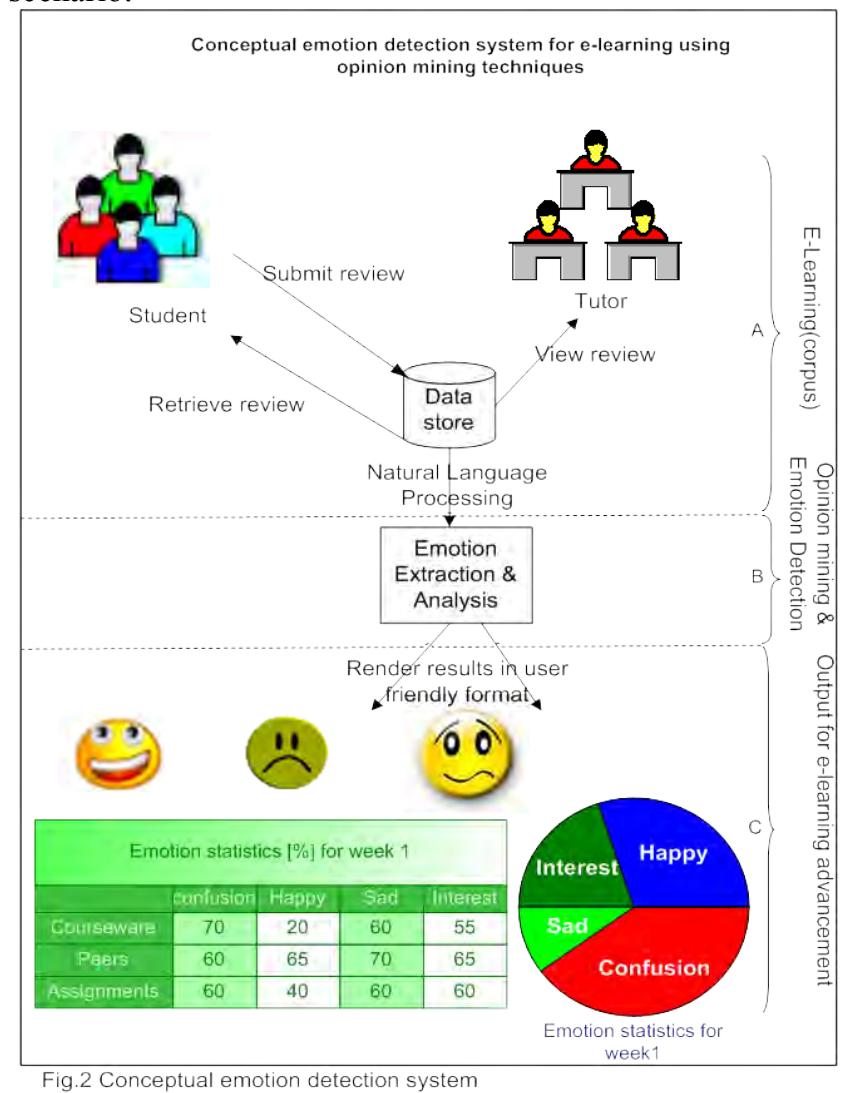

Fig.2 Conceptual emotion detection system

\section{A. E-Learning (corpus)}

The envisaged e-learning system shown in Fig.2 is an extension of Fig. 1 to show the practical application of an amalgam of e-learning, opinion mining and emotion detection. The test data (corpus) may be provided from student online journals in a blended course offering with time being allocated for seminars with a facilitator, group discussions as well as online exercises.

Currently, students are required to post a weekly online review to help them to write a reflection paper towards the end of the semester. The reflection paper is meant to assist students in assessing their own engagement and participation within the course. Furthermore, the tutor verifies that reviews are being posted and awards marks when this is done.

\section{B. Opinion mining \& Emotion Detection}

When data has been posted online by students, we intend to extract and analyse the text using opinion mining techniques. To achieve this, we will employ the General Text and Language Engineering Infrastructure (GATE). This is a tool specifically developed for research purposes involving language processing software. It has a word splitter, tokeniser, gazetteer and pos tagger. It will allow us to annotate text containing emotions as well as to perform quantitative analysis

Opinion mining research and emotion detection theory will be used for implementing a natural language processing (NLP) system. This will be the main focus of our research prototype. The major activities include lexical analysis and annotation.

\section{Output for e-learning advancement}

In the third section of Fig. 2, output for e-learning advancement, we present the output of our emotion detection system in the form of emoticons and graphs for analysis by the lecturer or unit coordinator.

In Fig. 3 we demonstrate an example of text annotated using GATE's visual environment. The GATE infrastructure provides a platform for developing, implementing and testing language processing modules. It can be used to split sentences using a splitter, tokenise them using a tokeniser and apply annotation rules. All these can be collectively classified as lexical analysis. Let us consider the statement, "This is an extremely exciting project". The splitter will split the preceding statement wherever a space is encountered. The tokeniser will then place each word into a token. In this case, there are six tokens. The next step will be to identify adjectives, verbs, subjects and objects by applying annotation rules. This analysis will enable us to identify emotions that are being exhibited. This will enable us to establish if the student is experiencing a positive (happy, engaged, excited), negative (sad, confused, frustrated) or neutral emotional experience. 


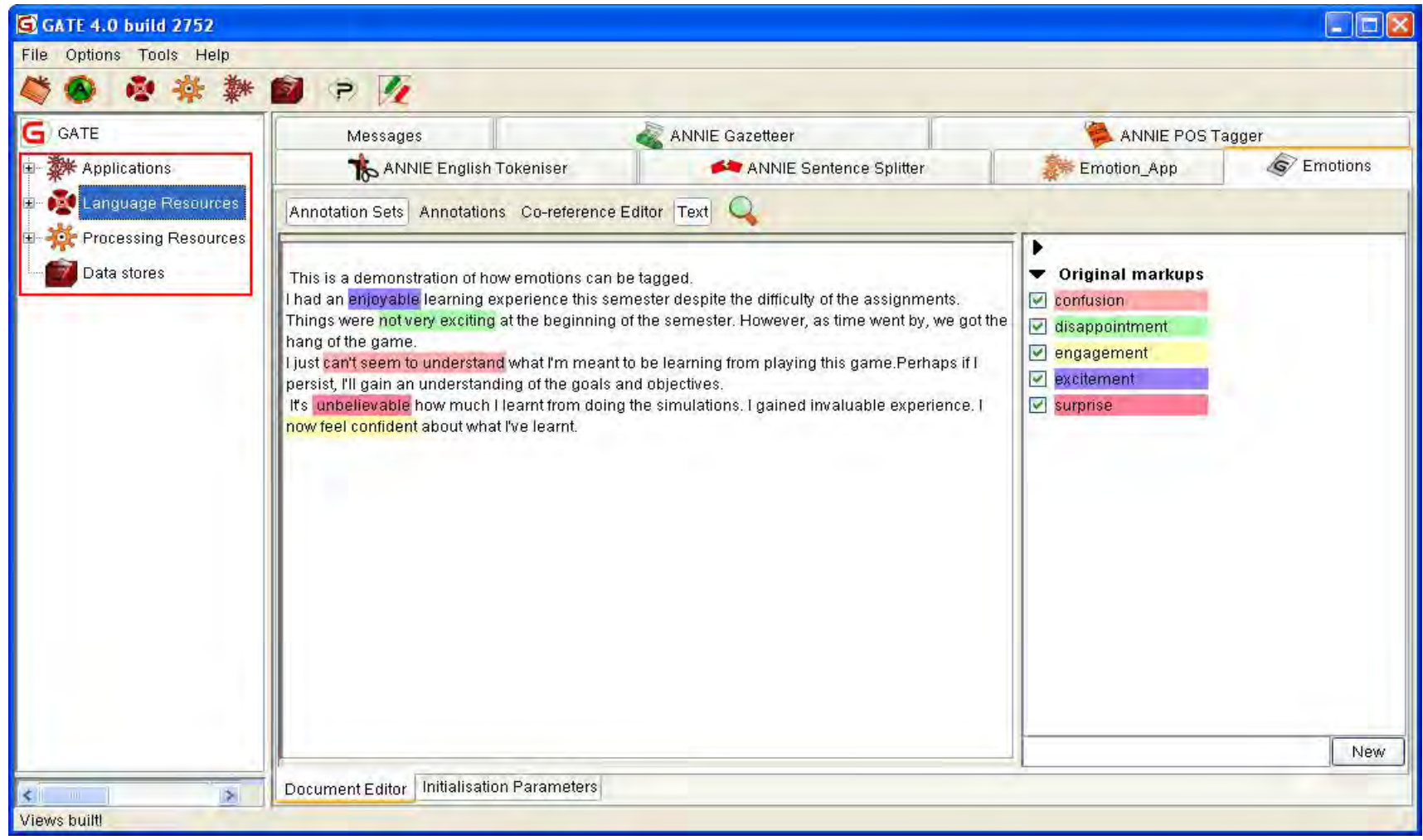

Fig.3 Overview of GATE's visual environment showing annotated text

\section{Contribution}

- Theoretical: We will identify emotions that are exhibited and their effect on the student's attainment of explicit learning objectives. This will open up an area that will attempt to bridge the gap between the business domain and e-learning domain. By changing the context of the solution from the former to the latter, it would be interesting to note how well the findings fit into the new context.

- Methodology: To develop an annotation style for the emotions involved in an e-learning environment. We want to develop a method that pinpoints specific clauses that contain opinions whose emotional content can be analysed.

- Empirical: A lot of research has already been carried out in the business domain to extract emotions using opinion mining techniques. We aim to establish a practical application of the knowledge areas within the e-learning domain. We will validate the feasibility of using existing emotion detection methods to assist student learning.

\section{E. Significance}

The research aims to provide a conceptual framework that can extract, analyse, and predict the emotion of learners (i.e. students). This will assist the lecturer to make wise decisions in order to tailor the teaching methods and processes. Furthermore, it will facilitate emotion detection in a natural and spontaneous fashion without using experimental instruments. This can help courseware designers and developers to present materials and project enthusiasm without direct visual feedback. A prototype may be used to detect fluctuations in emotion as learning progresses using opinion mining techniques.

\section{CONCLUSION}

In this paper, we have discussed the mile stones attained in opinion mining and e-learning. We note that researchers in these fields have also explored emotion theories and have integrated these into their studies. A new area of research significance in the e-learning domain has been revealed. A conceptual solution for an emotion detection and analysis system is presented. This will comprise a major component of our future research as we explore a junction on the frontiers of three research disciplines: Opinion mining, Elearning and Emotion detection. 


\section{REFERENCES}

[1] G. Woodhill, "Where is the learning in e-learning?: a critical analysis of e-learning industry," 2004.

[2] B. A. Goodman, F. N. Linton, R. D. Gaimari, J. M. Hitzeman, H. J. Ross, and G. Zarrella, "Using Dialogue Features to Predict Trouble During Collaborative Learning," User Modelling and User-Adapted Interaction, vol. 15, pp. 85-134, 2005.

[3] K. O'Reagan, "Emotion and e-learning," Journal of Asynchronous Learning Networks, vol. 7, pp. 7892, 2003.

[4] P. Chalfoun, S. Chaffar, and C. Frasson, "Predicting the Emotional Reaction of the Learner with a Machine Learning Technique," in Workshop on Motivational and Affective Issues in ITS. (G. Rebolledo-Mendez, E. Martinez-Miron, Eds), 2006, pp. 13-20.

[5] D. Song, H. Lin, and Z. Yang, "Opinion Mining in e-Learning System," in Network and Parallel Computing Workshops, 2007, pp. 788-792.

[6] S. McQuiggan, S. Lee, and J. Lester, "Early Prediction of Student Frustration," in in Proc. 2nd Intl. Conf. Affective Computing \& Intelligent Interaction (ACII 2007), 2007.

[7] W. Burleson and R. Picard, " Affective agents: Sustaining motivation to learn through failure and a state of stuck.," in Workshop of Social and Emotional Intelligence in Learning Environments, In 7th International Conference on Intelligent Tutoring System., 2004.

[8] R. Sharpe, G. Benfield, E. Lessner, and E. DeCicco, "Scoping Study for the Pedagogy strand of the JISC e-Learning Programme," 2005.

[9] I. Bessenyei, " Learning and Teaching in the Information Society. eLearning 2.0 and Connectivism. ," 2008.

[10] M. Prenksy, " Digital Natives, Digital Immigrants," in On the Horizon, 9(5), 2001, pp. 1-6.

[11] N. Hara and R. Kling, " Students' distress with a web-based distance education course. ," Information, Communication \& Society, vol. 3, pp. 557-559, 2000.

[12] A. Graesser, B. McDaniel, P. Chipman, A. Witherspoon, S. D'Mello, and B. Gholson, "Detection of emotions during learning with AutoTutor," in Proceedings of the 28th Annual Conference of the Cognitive Science Society, 2006, pp. 285-290.

[13] K. Leidelmeijer, Emotions: An Experimental Approach: Tilburg University Press, 1991.

[14] R. R. Kort and R. W. Picard, "An affective model of interplay between emotions and learning: Reengineering educational pedagogy -building a learning companion," in IEEE International Conference on Advanced Learning Technologies(ICALT'O1), 2001.

[15] M. Neji and M. B. Ammar, "Emotional eLearning
System " in Fourth International Conference on eLearning for Knowledge-Based Society, Bangkok, Thailand, 2007.

[16] M. Baptista-Nunes and M. McPherson, "No lectures on campus: can eLearning provide a better learning experience?," in IEEE International Conference on Advanced Learning Technologies., 2002.

[17] J. A. Russell, "Core affect and the psychological construction of emotion," Psychological Review, vol. 110, pp. 145-172, 2003.

[18] R. Sylwester, "How emotions affect learning," Educational Leadership, vol. 52, 1994.

[19] E. A. Linnenbrink, \& Pintrich, P. A., "Role of affect in change processing in academic contexts," in Motivation, emotion, and cognition: Integrative perspectives on intellectual functioning and development, R. J. S. D. Y. Dai, Ed. Mahwah, New Jersey: Lawrence Erlbaum Associates, 2004.

[20] B. Kort, Reilly, R., \& Picard, R., "An affective model of interplay between emotions and learning: Reengineering educational pedagogy — building a learning companion.," in Proceedings IEEE International Conference on Advanced Learning Technology: Issues, Achievements and Challenges, R. H. T. Okamoto, Kinshuk, \& J. P. Klus, Ed. Madison, Wisconsin: IEEE Computer Society, 2001, pp. 43-48.

[21] S. D'Mello, R. S. Taylor, and A. Graesser, "Monitoring Affective Trajectories during Complex Learning," in 29th Annual Meeting of the Cognitive Science Society, 2007.

[22] S. D'Mello, R. Picard, and A. Graesser, "Towards An Affect-Sensitive AutoTutor," IEEE Intelligent Systems, 2008.

[23] H. H. Binali, V. Potdar, and C. Wu, "A State Of The Art Opinion Mining And Its Application Domains," in Proceedings of ICIT09, 2009.

[24] A. Esuli and F. Sebastiani, "Determining Term Subjectivity and Term Orientation for Opinion Mining," in Proceedings the EACL 2006, 2006. 